\title{
Combined induction of rubber-hand illusion and out-of-body experiences
}

\section{Isadora Olivé * and Alain Berthoz}

Laboratoire de Physiologie de la Perception et de I'Action, UMR 7152, College de France, CNRS, Paris, France

\section{Edited by:}

Henk Barendregt, Radboud

University, Netherlands

\section{Reviewed by:}

Poppy Schoenberg, Radboud

University Nijmegen, Netherlands

Henk Barendregt, Radboud

University, Netherlands

\section{*Correspondence:}

Isadora Olivé, Laboratoire de Physiologie de la Perception et de I'Action, UMR 7152, Collège de France, CNRS, 11 Place Marcelin Berthelot, 75005 Paris, France. e-mail: isadora.olive@ college-de-france.fr
The emergence of self-consciousness depends on several processes: those of body ownership, attributing self-identity to the body, and those of self-location, localizing our sense of self. Studies of phenomena like the rubber-hand illusion (RHi) and out-of-body experience (OBE) investigate these processes, respectively for representations of a body-part and the full-body. It is supposed that $\mathrm{RHi}$ only target processes related to body-part representations, while OBE only relates to full-body representations. The fundamental question whether the body-part and the full-body illusions relate to each other is nevertheless insufficiently investigated. In search for a link between body-part and full-body illusions in the brain we developed a behavioral task combining adapted versions of the RHi and OBE. Furthermore, for the investigation of this putative link we investigated the role of sensory and motor cues. We established a spatial dissociation between visual and proprioceptive feedback of a hand perceived through virtual reality in rest or action. Two experimental measures were introduced: one for the body-part illusion, the proprioceptive drift of the perceived localization of the hand, and one for the full-body illusion, the shift in subjective-straightahead (SSA). In the rest and action conditions it was observed that the proprioceptive drift of the left hand and the shift in SSA toward the manipulation side are equivalent. The combined effect was dependent on the manipulation of the visual representation of body parts, rejecting any main or even modulatory role for relevant motor programs. Our study demonstrates for the first time that there is a systematic relationship between the body-part illusion and the full-body illusion, as shown by our measures. This suggests a link between the representations in the brain of a body-part and the full-body, and consequently a common mechanism underpinning both forms of ownership and self-location.

Keywords: self-consciousness, bodily self-consciousness, body ownership, self-location, visual, motor

\section{INTRODUCTION}

Philosophical and neuroscientific research aims to understand how different processes of using, being conscious of, and representing the physical body influence the institution of selfconsciousness (Eilan et al., 1998).

This question has recently been approached by illusion studies such as the rubber-hand illusion (RHi) and out-of-body experiences (OBE), which investigate body ownership, a process of attribution of self-identity to the body, and self-location, the spatial localization of the self, or the "I" of experience and behavior (Lenggenhager et al., 2007), which in health subjects is experienced within the body (Botvinick and Cohen, 1998; Ehrsson, 2007; Lenggenhager et al., 2007; Blanke and Metzinger, 2009; Tsakiris, 2010).

Both RHi and OBE target these processes through the establishment of a spatial dissociation between the localizations of the body and that of the self.

In RHi the spatial dissociation is restricted to the localization of a body-part and what some authors consider as sub-global aspect of self (Lenggenhager et al., 2007), a process named bodypart ownership. The global sense of self would be anchored to the stable full-body, and as such, would not be manipulated, and consequently self-location would not be investigated (Botvinick and Cohen, 1998; Ehrsson et al., 2004; Costantini and Haggard, 2007; Lenggenhager et al., 2007; Longo et al., 2008; Moseley et al., 2008).

The experimental mechanism behind RHi explores the synchronous stroking of a rubber-hand placed under the view of the subject in an anatomical plausible position, and one's own unseen hand. One could observe a mislocalization of the touch sensation on the rubber hand, and the consequent mislocalization or proprioceptive drift of the manipulated body-part toward the rubber hand. This proprioceptive drift is a commonly used objective measure for body-part illusion, and as such for body-part ownership (Tsakiris and Haggard, 2005).

On the other hand the study of the illusion of OBE investigate the process of self-location (Ehrsson, 2007; Lenggenhager et al., 2007) through the establishment of a dissociation in the spatial localization of the full-body and the sense of self (Lenggenhager et al., 2007; Blanke and Metzinger, 2009).

In OBE, the participant's back is stroked while he/she sees through a head-mounted display (HMD) the online stereoscopic recording of his/her full-body framed from the back in third person perspective (3PP). 
The mislocalization of the body toward its virtual proxy (Ehrsson, 2007; Lenggenhager et al., 2007) constitutes a commonly used objective measure for full-body illusion, and as such for full-body ownership and in some studies for self-location (Lenggenhager et al., 2007).

Illusions concerning a body-part and the full-body are induced by manipulation of the same parameters, encompassing the role of first-person perspective, the role of visual representation of the body-part or full-body, the role of proprioceptive cues of the body, and the role of localization of the visual representation of these proprioceptive cues (Ehrsson et al., 2004; Lenggenhager et al., 2007; Meyer, 2008; Petkova et al., 2011b).

Moreover, the neural substrates defined for body-part and fullbody illusions constitute of cortical structures, which in their majority accumulate dynamic information of body-part and fullbody, as well as processes of association of the sense of self to both bodily representations (Schubert et al., 1998; Shmuelof and Zohary, 2006; Felician and Romaiguere, 2008; Corradi-Dell'Acqua et al., 2009; Felician et al., 2009; Petkova et al., 2011a).

Such findings led some authors to postulate a tight link between body-part and full-body illusions (Lenggenhager et al., 2007). However, so far this subject is insufficiently explored and only recent data have demonstrated such a link specifically between body-part and full-body ownership (Petkova et al., 2011a).

Other central topics which are only partially explored are the potential role of sensory-only and motor cues in processes of body-part and full-body ownership (Georgieff and Jeannerod, 1998; Tsakiris et al., 2007; Tsakiris, 2010), and the generalization of body-part into full-body ownership (Tsakiris et al., 2006).

The role of motor programs in self-location also remains speculative. The two main hypothesis on the subject, one postulating a fundamental role for motor intentionality (Pacherie, 2008), and the other denying any necessary role for motor programs in general, but hypothesizing its modulatory role (Blanke and Metzinger, 2009), lack empirical support.

In this study we aim to unravel these questions of a putative link between body-part and full-body illusions and their consequence for processes of ownership and self-location, as well as their dependence on sensory-only or motor processes.

We adopted two experimental measures, the proprioceptive drift, an index of body-part illusion, and the shift in perceived subjective-straight-ahead (SSA), an index of full-body illusion.

The shift in SSA in relationship to the objective body midsagittal plane represents the body-centered frame of reference (Jeannerod and Biguer, 1989; Spidalieri and Sgolastra, 1997), indicating how individuals perceive this frame of reference and, by analogy, experience the objective localization of their full-bodies.

Our choice of experimental measures enable the investigation of a putative link between body-part and full-body illusions by analyzing the interplay between three basic spatial frameworks that the brain uses to localize the body in space: (1) the retinocentric frame of reference, which corresponds to the visual localization of the body-part or full-body, (2) the body-part frame of reference, investigated through the proprioceptive drift measure, and (3) the body-centered frame of reference, investigated through SSA, which together with (2) correspond to the proprioceptive and vestibular localization of the body (Graziano, 2001; Galati et al., 2010).
Spatial coordinate transformations relate these three frames of reference (Lacquaniti et al., 1995; Desmurget et al., 1998; Graziano, 2001; Avillac et al., 2007). A classical model of spatial coordinate transformations postulates that this process is decomposed in analytic steps ranging from the transform of retinotopic-to-eye centered frames of reference, eye-to-head, head-to-trunk, and trunk-to-arm frames of reference. Nevertheless, direct transform from eye-to-hand was reported, as well as the existence of neurons in ventral premotor cortex with body-part centered reference frames, which operate independent of retinotopy or eye position information (Desmurget et al., 1998; Graziano and Gross, 1998; Graziano, 2001; Avillac et al., 2007).

Moreover, spatial coordinate transformations are processed differently in the framework of motor programming or execution (Graziano, 2001). Gross visuomotor behaviors such as walking are programmed and executed in relation to the body-centered frame of reference, while focused movements such as reaching for an object are programmed in body-part centered frames of reference (Ventre et al., 1984; Filimon, 2010). Alternatively, motor programs may rely on a body representation based on relationships between body-part joint angles, dependent on proprioceptive information (Graziano, 2001; Martin et al., 2002).

In this study we hypothesize the existence of a tight link between body-part and full-body illusions based on evidence provided by behavioral and brain imaging studies mentioned above. Furthermore, we hypothesize that motor programs play no main role in the institution of this link between body-part and full-body illusions, because in previous studies RHi and OBE were elicited in absence of action execution (Botvinick and Cohen, 1998; Ehrsson, 2007; Lenggenhager et al., 2007).

According to our first hypothesis, we predict that manipulating visual representation of the body-part will induce a spatial coordinate transformation decomposed in analytic steps involving both body-centered and body-part centered frames of reference. Concerning the second hypothesis we predict that no differential pattern of transform related to the exact type of motor activity performed will be observed.

\section{MATERIALS AND METHODS EXPERIMENT 1 \\ IIlusions}

In this study, we aimed to induce two illusions, one adapted from the classical RHi, and the other adapted from the classical OBE. Our subjects were immersed in a virtual reality environment, where they saw in first-person perspective a stereoscopic image of their left hand acting upon or lying upon without grasping a mechanical piston. This virtual visual representation was displaced by about $30^{\circ} \mathrm{FOV}$ to the right of the physical handpiston ensemble. We considered that we replicate RHi illusion whenever the subject mislocalized the position of the physical hand-piston ensemble toward its virtual proxy at the right. Similarly, we replicate OBE whenever the subject mislocalized the position of the body-midline toward the right and in the orientation of the established spatial displacement between the visual representation of the hand-piston ensemble and its actual physical location. 


\section{Participants}

Fourteen healthy subjects, seven females and seven males (mean age $30.14 \pm 2.4$ ) with normal or corrected-to-normal vision, and no history of motor or neurological problems, volunteered to participate in the study. All subjects were verified right-handers by the Edinburgh Protocol. The research was approved by local ethics committee, and subjects signed an informed consent after being informed of the aims and procedures of the experiments.

\section{Experimental set-up and design}

Participants stood erect, wearing a pair of HMD (Z800 3D Visor e-Magin; $130^{\circ}-50^{\circ}$ field of view, FOV), with their head restrained by a headrest. Their left forearm lay in an armrest with adjustable level and angular position to the subject's best ergonomic position and avoiding complains of possible pain. The left hand of the subject was free upon the piston, and eventually grasped it with the left thumb lying on the tip-of-the-piston (TTP).

The final position of the TTP was fixed to $150^{\circ}$ FOV (field of view) for each participant and outside the scope of the HMD $\left(130^{\circ}-50^{\circ} \mathrm{FOV}\right)$. The HMD was connected to a Dell precision 390 desktop with Intel Duo Core 2 processor. Two video cameras (Wide LCD DCR-HC62 Sony) relayed the images to a Stereoscopic Multiplexer Configurator (version 0.5.2. Copyright ${ }^{\odot}$ 2005-2007 Peter Wimmer) and played online by a Stereoscopic Player (version 1.3.1. Copyright ${ }^{\odot}$ 2001-2007 Peter Wimmer).

The cameras were positioned to the right shoulder and at eyeslevel of each participant. The participant could see from a firstperson perspective his/her left forearm and piston with stereoscopic vision. The background of the recorded image was a black cloth to avoid interferences from the environmental, allocentric spatial cues in the task.

On the subject's right index finger was fixed a motion sensor (motion capture system flock of birds, FoB; Ascension Technology Corporation; Slave model $6 \mathrm{DFoB}$ ) fixed to record the kinematics of experimental pointing error measurements.

We developed a $2 \times 2$ factorial design with main factors presence or absence of vision, and presence or absence of action upon the piston. Four conditions were consequent, labeled as follows: vision*action execution (VA), the subject viewed his/her thumb pressing and releasing continuously the mechanical piston through the HMD; vision*no-action-execution (VNA), the subject viewed his/her hand lying upon without grasping the piston; no vision*action execution (NVA), the HMD was turned off and the subject continuously pressed and released the piston; and finally no vision*no-action-execution (NVNA), the HMD was blacked out and the subject's hand lying upon without grasping the piston. All four conditions lasted $40 \mathrm{~s}$. The order of the 8 experimental conditions ( 4 conditions $* 2$ pointing tasks) within each block was pseudo-randomized for each subject. A total of four trials for each condition grouped in four blocks were done with a total duration of $55 \mathrm{~min}$ and a pause as long as needed by each subject between each block.

In experimental and control conditions subjects were instructed via recorded commands transmitted through the HMD headphones to fix their visual attention on a virtual image of the hand-piston ensemble displayed on the HMD. All conditions were accompanied by auditory white noise.
After $40 \mathrm{~s}$, subjects were asked to execute one of two pointing tasks with a single brisk movement using their right index finger. No adjustments of the final position were allowed. The first, to point at the place of contact between the thumb and the tip of the mechanical piston (TTP task), indicating the perceived localization of the body-part engaged in this action. The second, to point to their body midline (SSA task), indicating the perceived localization of their full-body. Pointing tasks were executed with visual feedback of a frozen image of the subject's left hand grasping the piston, to avoid any visual guidance of the pointing movement. Subjects were instructed to retain the final pointing position until hearing the command "pause" on the headphones 5 s later.

\section{Statistical analysis}

The kinematics of the two pointing tasks TTP and SSA were collected by a motion capture system (FoB; Ascension Technology Corporation; Slave model $6 \mathrm{DFoB})$ recording the position $(x, y, z)$ and orientation (azimuth, elevation, roll) of a motion sensor fixed at the participant right index finger. The accuracy of the system was adjusted to $0.1 \mathrm{~mm}$ and all data were sampled by software specially developed to communicate with the FoB system.

Raw data collected by the FoB were pre-processed as arctangent data to provide measure of pointing error expressed in angles (degrees). Angular pointing errors were measured for each individual pointing task and averaged for each subject and conditions (four measures per subject per condition) via circular statistics software Oriana's 3.0.

For the TTP task, a first reference measurement was taken, indicating an ideal point where no illusion was involved (Figure 1A). This reference measurement was obtained for each subject and each block. The position of the TTP in $x$ and $y$ FoB coordinates were linked with the $x$ and $y$ FoB coordinates of last position of the participant's pointing finger $\left(\mathrm{TTP}^{\circ}\right)$. The actual pointing error was read as an angle from the azimuth value provided by the last position of the participant's pointing finger measured by the FoB. The experimental angle $\alpha^{\circ}$ was obtained by subtracting the reference measurement from the actual pointing angle. For clarity reasons, we standardized the reference angle at 180 degrees for all subjects, inducing a proportional adjustment of the experimental angles $\alpha^{\circ}$. Adjusted experimental angles $\alpha^{\circ}$ were analyzed as relative pointing errors with factorial circular ANOVA (Harrison et al., 1986; Harrison and Kanji, 1988), and labeled for each experimental condition as follow: $\alpha_{\mathrm{VA}}$ (visual feedback*action execution); $\alpha_{\mathrm{VNA}}$ (visual feedback $*$ no-actionexecution); $\alpha$ NVA (no-visual-feedback $*$ action execution); $\alpha_{\text {NVNA }}$ (no-visual-feedback $*$ no-action-execution).

For the SSA task, a referential position of perfect SSA was setthed at the perpendicular line at $90^{\circ}$ of the subjects' "between the eyes" $x$ and $y$ coordinates collected with FoB motion sensor when subjects were already placed in the set-up, and before each block (Figure 1B). An angle (degree) of actual pointing error was obtained linking this referential "between the eyes" coordinates and the last $x$ and $y$ FoB coordinates of the participant's pointing finger $\left(\mathrm{SSA}^{\circ}\right)$. The experimental angle $\beta^{\circ}$ was obtained by subtracting the angle of actual pointing error from the referential position of perfect SSA set at $90^{\circ}$. These angular 
measurements of error were processed as relative data with factorial circular ANOVA (Harrison et al., 1986; Harrison and Kanji, 1988), labeled for each experimental condition as follows: $\beta_{\mathrm{VA}}$ (visual feedback*action execution); $\beta_{\mathrm{VNA}}$ (visual feedback*noaction-execution); $\beta$ NVA (no-visual-feedback $*$ action execution); $\beta_{\text {NVNA }}$ (no-visual-feedback*no-action-execution).

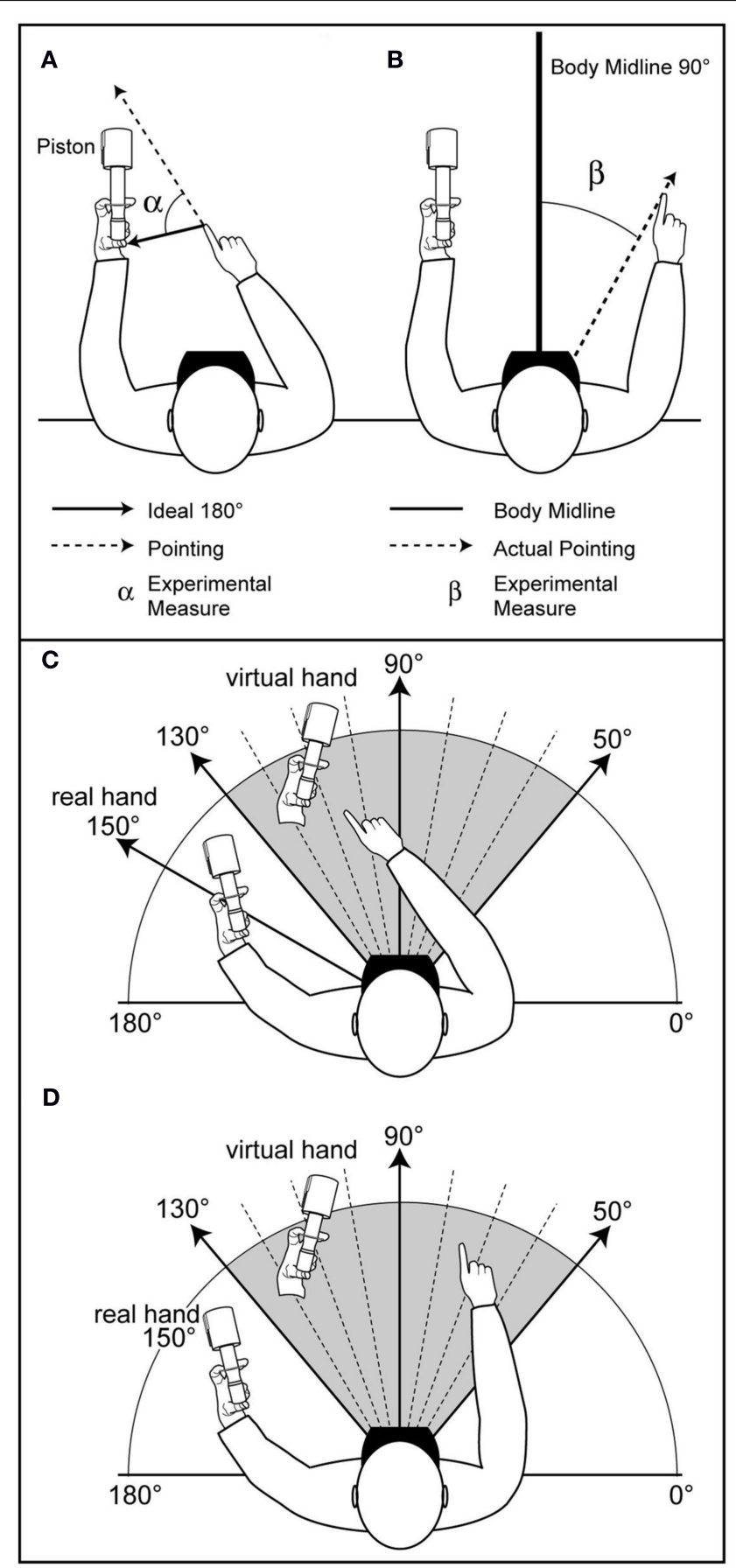

FIGURE 1 | Combined induction of adapted RHi and OBE effects. (A) Measurement of TTP pointing error. (B) Measurement of SSA pointing error. (C) RHi effect. (D) OBE effect.

\section{Results}

A significant drift of the perceived localization of the hand was observed related to the presence of visual feedback $\left(F_{1,52}=4.21\right.$; $p<0.05)$. Subjects presented a mean angle for the localization of the point of contact of the thumb and the TTP (ideally positioned at $180^{\circ}$ ) for condition visual feedback*action execution $\mathrm{TTP}_{\mathrm{VA}}=150.962^{\circ} \pm 6.968^{\circ}$ (circular mean $\pm 95 \%$ confidence interval $), \alpha_{V A}=29.038^{\circ}\left(\alpha=180^{\circ}-\mathrm{TTP}^{\circ}\right)$, and for visual feedback $*$ no-action-execution $\mathrm{TTP}_{\mathrm{VNA}}=153.26^{\circ} \pm 8.58^{\circ}$ $(\mathrm{cm} \pm 95 \% \mathrm{CI}), \alpha_{\mathrm{VNA}}=26.74^{\circ}$. Such mean angles drifted significantly by $10^{\circ}$ toward the virtual hand, in both acting and rest conditions, compared to both no-visual-feedback conditions: novisual-feedback $*$ action execution $\mathrm{TTP}_{\mathrm{NVA}}=160.214^{\circ} \pm 7.515^{\circ}$ $(\mathrm{cm} \pm 95 \% \mathrm{CI}), \alpha_{\mathrm{NVA}}=19.786^{\circ}$ and no-visual-feedback $*$ noaction-execution $\mathrm{TTP}_{\mathrm{NVNA}}=159.513^{\circ} \pm 8.275^{\circ}(\mathrm{cm} \pm 95 \% \mathrm{CI})$, $\alpha_{\text {NVNA }}=20.487^{\circ}$ (Table 1).

We analyzed the orientation of pointing errors for all conditions through calculation of concentration values, a measure of orientation analysis commonly used in circular statistics (Mello, 2005).

Concentration values $(r)$ close to 1 for all experimental and control conditions demonstrate that pointing errors significantly exhibited the same orientation left to right (Figure 1C), from the real hand and toward the virtual hand (Mello, 2005): $r_{\mathrm{VA}}=0.979$ (circular variance $=0.021$ and circular Standard Deviation $\left.=11.872^{\circ}\right), r_{\mathrm{VNA}}=0.968\left(\mathrm{cv}=0.032\right.$ and $\left.\operatorname{csdv}=14.618^{\circ}\right)$, $r_{\mathrm{NVA}}=0.975\left(\mathrm{cv}=0.025\right.$ and $\left.\operatorname{csdv}=12.802^{\circ}\right), \quad r_{\mathrm{NVNA}}=0.97$ $\left(\mathrm{cv}=0.03\right.$ and $\left.\operatorname{csdv}=14.099^{\circ}\right)$.

Post hoc analysis did not reveal any significant difference between the means of the two visual feedback factors, visual feedback*action execution (VA) and visual feedback*no-actionexecution (VNA). No main effect of action execution was observed $\left(F_{1,52}=0.03\right)$, nor an interaction between visual feedback and action execution $\left(F_{1,52}=0.18 ; p>0.05\right)$.

The same pattern of results found in mislocalization of the body-part (TTP tasks) was also observed for mislocalization of the full-body (SSA tasks). The main effect of visual feedback was significant $\left(F_{1,52}=6.5 ; p<0.05\right)$, revealing no main effect of action execution $\left(F_{1,52}<0.001 ; p>0.05\right)$, or interaction between visual feedback and action execution $\left(F_{1,52}<0.001 ; p>0.05\right)$. In the presence of a visual feedback, the mean drift angles for perceived full-body conditions with visual feedback*action

Table 1 | Mean experimental angles $\alpha$ (degree) for tip-of-the-piston (TTP) pointing error, and $\beta$ (degree) for subjective-straight-ahead (SSA) pointing error.

TTP

\begin{tabular}{lll}
\hline EXPERIMENT 1 & \\
VA & $29^{\circ}$ & $11.6^{\circ}$ \\
VNA & $26.74^{\circ}$ & $10^{\circ}$ \\
NVA & $19.79^{\circ}$ & $3.2^{\circ}$ \\
NVNA & $20.49^{\circ}$ & $2.56^{\circ}$ \\
EXPERIMENT 2 & & \\
DVA & $24.4^{\circ}$ & $2.4^{\circ}$
\end{tabular}


execution were $\mathrm{SSA}_{\mathrm{VA}}=78.405^{\circ} \pm 5.378^{\circ}$ (circular mean $\pm 95 \%$ $\mathrm{CI}), \beta_{\mathrm{VA}}=11.595^{\circ}\left(\beta=90^{\circ}-\mathrm{SSA}^{\circ}\right)$, and with visual feedback $*$ noaction-execution were $\mathrm{SSA}_{\mathrm{VNA}}=79.9^{\circ} \pm 5.064^{\circ}(\mathrm{cm} \pm 95 \% \mathrm{CI})$, $\beta_{\mathrm{VNA}}=10.1^{\circ}$. In conditions where no-visual-feedback was available, the mean angles were significantly smaller, suggesting no effect on no-visual-feedback $*$ action execution $\mathrm{SSA}_{\mathrm{NVA}}=$ $86.796^{\circ} \pm 7.684^{\circ}(\mathrm{cm} \pm 95 \% \mathrm{CI}), \beta_{\mathrm{NVA}}=3.204^{\circ}$, and on no-visualfeedback $*$ no-action-execution $\quad \mathrm{SSA}_{\mathrm{NVNA}}=87.442^{\circ} \pm 7.372^{\circ}$ $(\mathrm{cm} \pm 95 \% \mathrm{CI}), \beta_{\mathrm{NVNA}}=2.558^{\circ}$ (Table 1). Post hoc analysis did not reveal a significant difference between the means of the two levels of the visual feedback factor $\beta \mathrm{VA}$ and $\beta_{\mathrm{VNA}}\left(F_{1,26}<0.001\right.$; $p>0.05)$.

Concentration values $(r)$ very close to 1 for all conditions demonstrate that individual pointing errors robustly indicate the same orientation (Mello, 2005): $r_{\mathrm{VA}}=0.987$ $\left(\right.$ circular variance $=0.013$ and circular $\left.\mathrm{SD}=9.162^{\circ}\right), r_{\mathrm{VNA}}=0.989$ $\left(\mathrm{cv}=0.011\right.$ and $\left.\mathrm{csdv}=8.626^{\circ}\right), r_{\mathrm{NVA}}=0.974(\mathrm{cv}=0.026$ and $\left.\operatorname{csdv}=13.09^{\circ}\right), r_{\mathrm{NVNA}}=0.976\left(\mathrm{cv}=0.024\right.$ and $\left.\operatorname{csdv}=12.56^{\circ}\right)$.

The drift (Figure 1D) observed in the presence of a visual feedback was systematically oriented from left to right, and in the same orientation as observed in the TTP task, reproducing the introduced displacement between the real and virtual hands.

A circular-circular correlation analysis with jackknifed probabilities was run with circular statistics software Oriana 4.01, demonstrating that those participants that show the largest drift on the TTP task are also those that show the largest drift in the SSA task, $r=0.292, p<0.05$.

\section{EXPERIMENT 2 \\ Test}

In Experiment 1 we observed no difference between conditions VA and VNA, suggesting that the presence or absence of proprioceptive information about the body, in the present study the force feedback, constitute no necessary role in emergence of both RHi and OBE, and as such, of body-part ownership and selflocation. Here, in our second study, we reproduce the standard test developed in previous studies in order to demonstrate the essential role of multisensory stimulation upon the body for eliciting of RHi and OBE. We applied a delay in the delivery of visual feedback, but only for the main experimental condition VA, with the presence of a visual feedback and an action execution. We created, in this way, the condition VDA, meaning visual with delay in presence of action execution. It is assumed that whether RHi and OBE are dependent on multisensory processes, the introduction of delay will reduce or totally abolish these illusions.

\section{Participants, experimental set-up and design, data collection and analysis}

Experiment 2 was carried out directly after Experiment 1 in the same session. In Experiment 2 we used the same participants, procedures, and data collection and analysis as in Experiment 1. The main difference is the utilization of two visual delay lines (Ovation Systems ltd.) in the experimental set-up, enabling the manipulation of synchronic delivery of visual feedback with the HMD. In condition VDA the delay was set at $250 \mathrm{~ms}$.

\section{Results}

In the TTP task, a significant difference was observed between the introduction of a delay of $250 \mathrm{~ms}$ in the display of the visual feedback and the onset of the action execution $\left(F_{1,26}=7.048 ; p<0.05\right)$. It induced a decrease of about $5^{\circ}$ between circular means in the experimental condition visual feedback*action execution $\mathrm{TTP}_{\mathrm{VA}}=150.962^{\circ} \pm 6.968^{\circ}$ (circular mean $\pm 95 \% \mathrm{CI}), \alpha_{\mathrm{VA}}=29.038^{\circ}\left(\alpha=180^{\circ}-\mathrm{TTP}_{\mathrm{VA}}\right)$ and the delayed control condition visual feedback $*$ action execution $\mathrm{TTP}_{\mathrm{DVA}}=155.623^{\circ} \pm 7.888^{\circ}(\mathrm{cm} \pm 95 \% \mathrm{CI}), \alpha_{\mathrm{DVA}}=24.377^{\circ}$ (Table 1). In this way, with the introduction of a delay, the localization was closer to the real piston than to the visual representation, as observed in the condition with near-to-perfect synchronicity.

In the SSA task again a mean effect was observed, by introducing a delay of $250 \mathrm{~ms}$ in the visual feedback $\left(F_{1,26}=4.28\right.$; $p<0.05$ ), provoking a practical disappearance of the spatial drift: $O S A_{V A}=78.405^{\circ} \pm 5.378^{\circ}$ (circular mean $\pm 95 \% \mathrm{CI}$ ), $\beta_{\mathrm{VA}}=11.595^{\circ}\left(\beta=90^{\circ}-\mathrm{OSA}^{\circ}\right)$, and $\mathrm{OSA}_{\mathrm{DVA}}=87.364^{\circ} \pm 7.373^{\circ}$ $(\mathrm{cm} \pm 95 \% \mathrm{CI}) ; \beta_{\mathrm{DVA}}=2.636^{\circ}$.

A circular-circular correlation analysis with jackknifed probabilities was run with circular statistics software Oriana 4.01, demonstrating that those participants that show the most significant decrease in illusion efficiency on the TTP task are also those that show the most significant decrease in illusion efficiency on the SSA task, $r=0.481, p<0.05$.

\section{DISCUSSION}

In this study, we aimed to unravel a putative link between bodypart and full-body illusions to redefine our understanding of associated processes of body ownership and self-location. Moreover, we investigated the role of sensory-only and motor mechanisms in this putative link.

To test this, we developed for the first time a protocol combining an adapted version of the RHi and of the OBE illusion. We immersed our healthy subjects in a virtual environment, displaying a stereoscopic representation of their left hand in rest or acting state against a black background. We worked with two experimental measures, one indicating the perceived localization of the body-part, the proprioceptive drift of the left hand, commonly used in RHi (Tsakiris and Haggard, 2005), and we introduced a new measure of perceived localization of the full-body, the shift of the SSA (Jeannerod and Biguer, 1989).

Our results indicate that the visual feedback of the experimental left hand produced a proprioceptive drift of hand position and a shift of the SSA of an equivalent amount and oriented toward the right, thus, toward the visual, virtual representation of this hand.

Our results suggest the existence of a tight link between bodypart and full-body illusions in the brain, which most likely are underpinned by a mechanism of spatial coordinate transformations, decomposed in analytic steps involving coordinated recalibration of both trunk or body-centered and hand- or body-part centered frames of reference (Desmurget et al., 1998). Our findings seem to be, in this way, in accordance with models of body-part coordination during prism adaptation, which demonstrate that trunk-on-arm body angles are processed as a unit (Martin et al., 2002). 
Such interpretation of our findings seems to point to a central role of the posterior parietal cortex (PPC) in our observed combined effect. More specifically, they seem to define an unequivocal role for the superior parietal lobe (SPL; Lacquaniti et al., 1995), as well as the intraparietal sulcus (IPS; Avillac et al., 2007), areas engaged in representation of the body in space, operating through population coding and merging different frames of reference, from retinotopic to body-centered and body-part centered frames of reference (Desmurget et al., 1998; Graziano, 2001).

To these areas we may add the inferior parietal lobe structures, the supramarginal gyrus (SMG) and the angular gyrus (ANG), which were previously related to processing of both body-part and global-body representations (Felician and Romaiguere, 2008; Felician et al., 2009). This accumulative role is also observed for IPS, which processes both body-part and global-body representations, and the mereological relationship between both of them (Schubert et al., 1998; Shmuelof and Zohary, 2006; CorradiDell'Acqua et al., 2009; Hodzic et al., 2009).

Together with the superior temporal gyrus (STG), SMG, ANG, and IPS constitute the temporoparietal junction (TPJ; Macaluso, 2000; Blanke et al., 2004). Neuronal damage in the TPJ induces spatial hemineglect, a disorder associated with shift in SSA (Rushmore et al., 2006). As such, TPJ seems to constitute a crucial node where manipulation of body-part representations would affect full-body representations, most likely through merging of body-part and body-centered frames of reference.

No direct and exclusive transform of visual frames of reference to body-part centered frames of reference seems to be engaged in our observed combined recalibration of body-part and full-body representations. Nevertheless, one very recent model, targeting the generalization of ownership from body-part to the full-body, demonstrated that this process may involve the population of neurons at ventral premotor cortex with body-part centered frames of reference (Graziano and Gross, 1998; Petkova et al., 2011a).

Whether such a model may apply to our present results is uncertain, mostly because of several differences between this study and ours. First, they targeted ownership processes which, as we will discuss in detail later, we are not sure to have manipulated. Furthermore, they investigated ownership through manipulation of synchronicity factors in applied tactile stimulation upon the subject and the virtual bodies, while in our study our combined effect is obtained independently of stimulation upon the body. Finally, their observed results were strongly correlated with the position of the stimulated body-part in relation to the full-body, and only when they were attached a strong illusion of ownership aroused. In our study, the body-part is by default observed in isolation, exhibiting no direct relation to the full-body.

However, the premotor cortex exhibits strong direct reciprocal connections with SMG, ANG (Rushworth et al., 2006; Koch et al., 2010), and IPS (Uddin et al., 2010). As previously suggested in the literature, these activations observed in PMv may represent a final processing step of both body-part and full-body illusions, when they become conscious (Ehrsson et al., 2004; Petkova et al., 2011a).

Our combined effect was due to manipulation of visual information alone, exhibiting no main or modulatory role for motor programs in it. Our results are in line with models of spatial coordinate transformations that postulate a basic dichotomy between spatial frameworks coordinating gross visuomotor behavior and focalized movements such as reaching and grasping (Ventre et al., 1984; Filimon, 2010). If we were to observe an effect of motor programs in our experiments, once we only manipulated a focalized movement of pressing and releasing continuously the piston, most likely only body-part centered frames of reference would be manipulated, inducing an isolated proprioceptive drift of the acting hand. Likewise it would be manipulated differently from the SSA, with TTP and SSA measures exhibiting no correlation. Alternatively, no main effect of vision would be observed, according to the hypothesis that motor programs are coordinated by relationships between joint angles, relying on proprioceptive cues only, and not involving spatial frames of reference (Graziano, 2001). In this way, we can conclude that our findings support models postulating the necessary and sufficient role of sensory mechanisms for the emergence of body ownership (Tsakiris et al., 2007; Blanke and Metzinger, 2009) and do not support models relying on the central role of motor programs (Georgieff and Jeannerod, 1998).

Moreover, our results do not agree with the postulate according to which intentional motor acts would generalize body-part ownership into a full-body ownership (Tsakiris et al., 2006). Our observed spatial displacement of the full-body measure was not influenced by action execution, being also observed in condition VNA, where SSA measures of full-body displacement were highly significant without any action execution.

Finally, our results support previous studies hypothesizing that motor programs play no necessary role in the processing of selflocation (Blanke and Metzinger, 2009), and further suggest that motor programs do not play any modulatory role in its experience. In consequence, most likely motor programs play no role in the engagement of body parts in defining of self-location.

Nevertheless, our experimental measures are restricted to objective spatial drift observations, a limitation imposed by the pseudo-random experimental design, and may represent a drawback for evaluating self-related processes, which are normally investigated through subjective measures such as questionnaire answers (Botvinick and Cohen, 1998; Ehrsson, 2007; Lenggenhager et al., 2007). Moreover, there is a current debate concerning the validity of drift measures for evaluating self-related processes, or more specifically, body ownership processes (Rohde et al., 2011). Nevertheless, this debate is based on the lack of systematic correlation between drift measures and the factor synchronicity of application of tactile stimulation upon the body, which was found to correlate significantly with subjective reports of ownership illusion as evaluated by questionnaire answers (Rohde et al., 2011). As such, this interpretation is only valid from the perspective of classical models of ownership experiences, both for body-part and full-body illusions, which postulate the necessary role of synchronous tactile stimulation upon the body (Botvinick and Cohen, 1998; Ehrsson, 2007; Lenggenhager et al., 2007). On the other hand, it does not apply to more recent models demonstrating that ownership illusions can be elicited without any type of stimulation upon the body (Slater et al., 2010). In our present study, we observed a combined effect in presence or absence of stimulation related to the body, thus, whenever the subject acted upon 
the piston, or the subject's hand rested upon the piston. In both conditions, TTP and SSA tasks exhibited measures of spatial drift of equal amount and orientation. In this way, our results seem to support models of body ownership, postulating that tactile stimulation applied upon the body does not constitute a necessary condition for experiencing ownership illusions (Slater et al., 2010). As such, in spite of lacking subjective report data, and of the fact that we worked with drift or shift measures, the possibility remains open that in our study self-related processes, such as body ownership and self-location, were also manipulated.

One must notice, though, that in Experiment 2, we observed that introduction of a multisensory conflict operationalized by the introduction of a temporal delay in delivery of visual representation of the performed action, induced a decay or abolishment of both illusions, a result in agreement with classical models of body ownership and self-location (Botvinick and Cohen, 1998; Ehrsson, 2007; Lenggenhager et al., 2007). Nevertheless, we interpret these results as evidence that both illusions are dependent on similarity between experienced real and virtual events, a condition that is only rendered evident by manipulating the delivery time factor. Most likely, the spatial drift of the tactile sensation would bring into awareness the actual basic component that allows emergence of both illusions, namely processes of spatial coordinate transformations relating visual frames of reference to body-part and body-centered frames of reference. These processes are independent of and precede all stimulation applied on the body.

We would like to conclude this discussion calling attention to the fact that in the present study we only manipulated visual and proprioceptive, or force sensation, cues. Most likely, the manipulation of vestibular cues, which was demonstrated to modulate or induce both RHi (Lopez et al., 2010) and OBE (Blanke et al., 2004) would enhance our understanding of neurocognitive principles

\section{REFERENCES}

Avillac, M., Ben Hamed, S., and Duhamel, J. R. (2007). Multisensory integration in the ventral intraparietal area of the macaque monkey. J. Neurosci. 27, 1922-1932.

Blanke, O., Landis, T., Spinelli, L., and Seeck, M. (2004). Out-of-body experience and autoscopy of neurological origin. Brain 127, 243-258.

Blanke, O., and Metzinger, T. (2009). Full-body illusions and minimal phenomenal selfhood. Trends Cogn. Sci. (Regul. Ed.) 13, 7-13.

Botvinick, M., and Cohen, J. (1998). Rubber hands 'feel' touch that eyes see. Nature 391, 756.

Corradi-Dell'Acqua, C., Tomasino, B., and Fink, G. R. (2009). What is the position of an arm relative to the body? Neural correlates of body schema and body structural description. J. Neurosci. 29, 4162-4171.

Costantini, M., and Haggard, P. (2007). The rubber hand illusion: sensitivity and reference frame for body ownership. Conscious. Cogn. 16, 229-240. underpinning the link between body-part and full-body illusions observed in the present study.

\section{CONCLUSION}

In the present study we demonstrated for the first time a tight link between body-part and full-body illusions, showing that manipulating the visual representation of a body-part observed through virtual reality constitutes a sufficient factor to induce a drift in perceived body-part and full-body. Both were mislocalized outside bodily boundaries, and the mislocalization assumed equivalent amounts and orientation from the real body and towards its virtual proxy. The present effect was obtained in either the presence or absence of stimulation on the body, suggesting that it may not constitute a necessary nor sufficient factor for instituting bodypart ownership or self-location. Finally, no role was found for motor programs in the combined manipulation of body-part and full-body illusion measures, suggesting that they play no main or modulatory role in ownership or self-location processes. We interpret our findings as evidence for the role of a common multisensory process of spatial representation coordinating retinotopic, body-centered and body-part centered frames of reference for localizing the body in space, driven by body-part visual representation manipulation. As such, our findings may cast a new light on understanding body-related neurological disorders, providing evidence that disorders related so far with body-part representations, such as somatoparaphrenia, are indeed accompanied by a full-body component. Further studies are needed to confirm this hypothesis.

\section{ACKNOWLEDGMENTS}

We would like to thank Yves Dupraz, Michael Zugaro, Cuong Quam Pham, Eve Dupierrix, and Christine Esslinger for help with technical assistance.

Filimon, F. (2010). Human cortical control of hand movements: parietofrontal networks for reaching, grasping, and pointing. Neuroscientist 16, 388-407.

Galati, G., Pelle, G., Berthoz, A. and Committeri, G. (2010). Multiple reference frames used by the human brain for spatial perception and memory. Exp. Brain Res. 206, 109-120.

Georgieff, N., and Jeannerod, M. (1998). Beyond consciousness of external reality: a "who" system for consciousness of action and selfconsciousness. Conscious. Cogn. 7, 465-477.

Graziano, M. S. (2001). Is reaching eye-centered, body-centered, handcentered, or a combination? Rev. Neurosci. 12, 175-185.

Graziano, M. S., and Gross, C. G. (1998). Spatial maps for the control of movement. Curr. Opin. Neurobiol. 8, 195-201.

Harrison, D., and Kanji, G. (1988). The development of analysis of variance for circular data. J. Appl. Stat. 15, 197-223.

Harrison, D., Kanji, G., and Gadsden, R. (1986). Analysis of variance for circular data. J. Appl. Stat. 13, 123-138.

Hodzic, A., Kaas, A., Muckli, L., Stirn, A., and Singer, W. (2009). Distinct cortical networks for the detection and identification of human body. Neuroimage 45, 1264-1271.

Jeannerod, M., and Biguer, B. (1989). sented space. Rev. Neurol. (Paris) 145, 635-639.

Koch, G., Cercignani, M., Pecchioli, C., Versace, V., Oliveri, M., Caltagirone, C., Rothwell, J., and Bozzali, M. (2010). In vivo definition of parieto-motor connections involved in planning of grasping movements. Neuroimage 51, 300-312.

Lacquaniti, F., Guigon, E., Bianchi, L., Representing spatial information for limb movement: role of area 5 in the monkey. Cereb. Cortex 5, 391-409. Egocentric reference and repreFerraina, S., and Caminiti, R. (1995). 
Lenggenhager, B., Tadi, T., Metzinger, T., and Blanke, O. (2007). Video ergo sum: manipulating bodily self-consciousness. Science 317, 1096-1099.

Longo, M. R., Schuur, F., Kammers, M. P., Tsakiris, M., and Haggard, P. (2008). What is embodiment? A psychometric approach. Cognition 107, 978-998.

Lopez, C., Lenggenhager, B., and Blanke, O. (2010). How vestibular stimulation interacts with illusory hand ownership. Conscious. Cogn. 19, 33-47.

Macaluso, E. (2000). Unimodal and Multimodal Mechanisms of Spatial Attention in the Human Brain. Ph.D. thesis, University College of London, London.

Martin, T. A., Norris, S. A., Greger, B. E., and Thach, W. T. (2002). Dynamic coordination of body parts during prism adaptation. J. Neurophysiol. 88, 1685-1694.

Mello, C. (2005). Statistiques circulaires et utilisations en psychologie. Tutorials Quant. Methods Psychol. 1, 11-17.

Meyer, K. (2008). How does the brain localizes the self? Science.

Moseley, G. L., Olthof, N., Venema, A., Don, S., Wijers, M., Gallace, A., and Spence, C. (2008). Psychologically induced cooling of a specific body part caused by the illusory ownership of an artificial counterpart. Proc. Natl. Acad. Sci. U.S.A. 105, 13169-13173.
Pacherie, E. (2008). The phenomenology of action: a conceptual framework. Cognition 107, 179-217.

Petkova, V. I., Bjornsdotter, M., Gentile, G., Jonsson, T., Li, T. Q., and Ehrsson, H. H. (2011a). From part- to wholebody ownership in the multisensory brain. Curr. Biol. 21, 1118-1122.

Petkova, V. I., Khoshnevis, M., and Ehrsson, H. H. (2011b). The perspective matters! Multisensory integration in ego-centric reference frames determines full-body ownership. Front. Psychol. 2:35. doi:10.3389/fpsyg.2011.00035

Rohde, M., Di Luca, M., and Ernst, M. O. (2011). The rubber hand illusion: feeling of ownership and proprioceptive drift do not go hand in hand. PLoS ONE 6, e21659. doi:10.1371/journal.pone.0021659

Rushmore, R. J., Valero-Cabre, A., Lomber, S. G., Hilgetag, C. C., and Payne, B. R. (2006). Functional circuitry underlying visual neglect. Brain 129, 1803-1821.

Rushworth, M. F., Behrens, T. E., and Johansen-Berg, H. (2006). Connection patterns distinguish 3 regions of human parietal cortex. Cereb. Cortex $16,1418-1430$.

Schubert, T., Von Cramon, D. Y., Niendorf, T., Pollmann, S., and Bublak, P. (1998). Cortical areas and the control of selfdetermined finger movements: an fMRI study. Neuroreport 9, 3171-3176.
Shmuelof, L., and Zohary, E. (2006) A mirror representation of others' actions in the human anterior parietal cortex. J. Neurosci. 26, 9736-9742.

Slater, M., Spanlang, B., Sanchez-Vives, M. V., and Blanke, O. (2010) First person experience of body transfer in virtual reality. PLoS ONE 5, e10564. doi:10.1371/journal.pone.0010564

Spidalieri, G., and Sgolastra, R. (1997). Psychophysical properties of the trunk midline. J. Neurophysiol. 78, 545-549.

Tsakiris, M. (2010). My body in the brain: a neurocognitive model of body-ownership. Neuropsychologia 48, 703-712.

Tsakiris, M., and Haggard, P. (2005). The rubber hand illusion revisited: visuotactile integration and selfattribution. J. Exp. Psychol. Hum. Percept. Perform. 31, 80-91.

Tsakiris, M., Prabhu, G., and Haggard, P. (2006). Having a body versus moving your body: how agency structures body-ownership. Conscious. Cogn. 15, 423-432.

Tsakiris, M., Schutz-Bosbach, S., and Gallagher, S. (2007). On agency and body-ownership: phenomenological and neurocognitive reflections. Conscious. Cogn. 16, 645-660.

Uddin, L. Q., Supekar, K., Amin, H., Rykhlevskaia, E., Nguyen, D. A., Greicius, M. D., and Menon,
V. (2010). Dissociable connectivity within human angular gyrus and intraparietal sulcus: evidence from functional and structural connectivity. Cereb. Cortex 20, 2636-2646.

Ventre, J., Flandrin, J. M., and Jeannerod, M. (1984). In search for the egocentric reference. A neurophysiological hypothesis. Neuropsychologia 22, 797-806.

Conflict of Interest Statement: The authors declare that the research was conducted in the absence of any commercial or financial relationships that could be construed as a potential conflict of interest.

Received: 16 September 2011; accepted: 01 March 2012; published online: 31 May 2012.

Citation: Olivé I and Berthoz A (2012) Combined induction of rubberhand illusion and out-of-body experiences. Front. Psychology 3:128. doi: 10.3389/fpsyg.2012.00128

This article was submitted to Frontiers in Cognition, a specialty of Frontiers in Psychology.

Copyright (c) 2012 Olivé and Berthoz. This is an open-access article distributed under the terms of the Creative Commons Attribution Non Commercial License, which permits non-commercial use, distribution, and reproduction in other forums, provided the original authors and source are credited. 